\section{Abstract}

20

21

22

23

24

\title{
Understanding Taxi Travel Patterns
}

\author{
Hua Cai ${ }^{12^{*}}$, Xiaowei Zhan ${ }^{3} \mathrm{Ji}_{\mathrm{Zhu}}{ }^{4}$, Xiaoping $\mathrm{Jia}^{5}$, Anthony S.F. Chiu ${ }^{6}, \mathrm{Ming}_{\mathrm{Xu}} \mathrm{u}^{78^{*}}$
}

1. School of Industrial Engineering, Purdue University, West Lafayette, Indiana, USA

2. Division of Environmental and Ecological Engineering, Purdue University, West Lafayette, Indiana, USA

3. Quantitative Biomedical Research Center, Department of Clinical Sciences, Center for the Genetics of Host Defense, University of Texas Southwestern Medical Center, Dallas, TX

4. Department of Statistics, University of Michigan, Ann Arbor, Michigan, USA

5. School of Environment and Safety Engineering, Qingdao University of Science and Technology, Qingdao, China

6. Department of Industrial Engineering, De La Salle University, Manila, Philippines

7. School of Natural Resources and Environment, University of Michigan, Ann Arbor, Michigan, USA

8. Department of Civil and Environmental Engineering, University of Michigan, Ann Arbor, Michigan,

* Corresponding authors:

Email: huacai@purdue.edu; Tel: +1-765-494-7701; Fax: +1-765-494-7704

Email: mingxu@umich.edu; Tel: +1-734-763-8644; Fax: +1-734-936-2195

\section{USA}

Taxis play important roles in modern urban transportation systems, especially in mega cities. While providing necessary amenities, taxis also significantly contribute to traffic congestion, urban energy consumption, and air pollution. Understanding the travel patterns of taxis is thus important for addressing many urban sustainability challenges. Previous research has primarily focused on examining the statistical properties of passenger trips, which include only taxi trips occupied with passengers. However, 
unoccupied trips are also important for urban sustainability issues because they represent potential opportunities to improve the efficiency of the transportation system. Therefore, we need to understand the travel patterns of taxis as an integrated system, instead of focusing only on the occupied trips. In this study we examine GPS trajectory data of 11,880 taxis in Beijing, China for a period of three weeks. Our results show that taxi travel patterns share similar traits with travel patterns of individuals but also exhibit differences. Trip displacement distribution of taxi travels is statistically greater than the exponential distribution and smaller than the truncated power-law distribution. The distribution of short trips (less than 30 miles) can be best fitted with power-law while long trips follow exponential decay. We use radius of gyration to characterize individual taxi's travel distance and find that it does not follow a truncated power-law as observed in previous studies. Spatial and temporal regularities exist in taxi travels. However, with increasing spatial coverage, taxi trips can exhibit dual high probability density centers.

\section{Keywords}

Taxi travel pattern; human mobility dynamics; vehicle trajectory

\section{Introduction}

Understanding the dynamics of human mobility is critical to many fields such as epidemics $[1,2]$, transportation [3, 4], urban planning [5], and genetics [6]. Recent development of information and communications technology (ICT) has significantly improved our ability to collect, store, and analyze large-scale datasets, which enables studying human mobility at a wide range of spatial and temporal scales [7]. Analyzing statistical properties of bank notes circulation as a proxy of human movement, Brockmann et al. (2006) observed that human travel distance exhibits a power law distribution and human travel trajectories may be approximated as Lévy flights (heavy tailed random walk) [8]. This observation was confirmed by Rhee et al. (2011) using Global Positioning System (GPS) traces collected from volunteers, showing non-negligible probability of high displacement trips and long pause-time between trips [9]. Despite the randomness indicated by Lévy flight models, using cell phone traces, Gozález et al. 
49 (2008) discovered that strong spatial and temporal regularities exist for human trajectories —individuals

50 have a tendency to frequently revisit a few locations [10]. To explain the observed scaling properties and

51 regularities, Song et al. (2010) proposed a combination of two mechanisms, exploration and preferential

52 return, for modeling human mobility [11]. It is worth noting that data used in these studies include

53 trajectories generated from different transportation modes (walking, driving, public transportation, and

54 flying etc.). Yan et al. (2013) argued that transportation modes affect aggregated travel patterns and

55 displacements from single mode transportation should follow an exponential distribution instead of power

56 law [12].

Vehicular travel is one important transportation mode that has implications to many societal

58

59

60

61

62

63

64

challenges [13]. Due to the privacy concerns related to private vehicle travel data, most studies on examining vehicle travel patterns so far are based on taxi trajectories captured by GPS. Despite having different travel needs compared to private vehicles, taxis can be viewed as floating sensors in a city that provide invaluable information on mobility dynamics, traffic conditions, and epidemic spreading risks. In addition, compared to other datasets used in studying human mobility dynamics such as the movement of bank notes [8], smart card data [14], and mobile phone records [10], GPS traces of taxis usually provide more detailed information with finer spatial and temporal resolutions and larger sampling size.

Previous studies on taxi travel patterns have been focused on extracting passenger trips to understand passenger travel patterns [3,15-17]. Peng et al. (2012) characterized taxi passenger trips for different travel needs [16]. Jiang et al. (2009) verified the scaling properties of taxi passenger-trip length and suggested that such property is attributed to the underlying street topology [3]. Liang et al. (2012) found that passenger trip displacements of taxis can be better approximated with exponential distributions instead of power law, possibly due to high costs associated with long taxi trips [17]. However, passenger trips are not representative of how taxis travel. In many cities, taxis always cruise around between passenger trips $[18,19]$. In some countries such as China, taxi drivers possess the vehicle throughout the day, sharing similar commuting patterns with private vehicles (i.e., returning home after work and then 
74 driving out for business the next day). To date, limited attentions have been paid to the patterns of taxi 75 travels as a whole.

Understanding taxi travel patterns can also contribute to addressing several urban sustainability

77 challenges. In particular, taxis are important components of the public transportation sector [13],

78 providing flexible and convenient mobility solutions for urban residents. Because they are on the road all

79 the time, taxis also contribute significantly to traffic congestion, energy consumption, and air pollution in

80 the cities [20]. In addition, taxis in many countries are likely to be early adopters of emerging

81 technologies (e.g., electric vehicles, connected and autonomous vehicles) and business models (e.g., Uber)

$82[21,22]$. Therefore, it is important to understand the travel patterns of taxis, taking into account the taxi

83 travels both with and without passengers. This research aims to fill this gap by examining taxi trajectory

84 data holistically to understand how taxi travel patterns are similar or different from those of individuals

85 and private vehicles. Results of this research can help guide traffic modeling, transportation planning,

86 urban planning, and infrastructure development.

\section{2. Methods}

88 This study examines vehicle trajectory data of taxis in Beijing to study taxi travel patterns. The data are analyzed from the perspective of characteristic travel length (radius of gyration), distribution of trip distance, and spatial density distribution in each taxi's intrinsic reference frame.

92 The dataset used in this study contains trajectory data of 11,880 taxis in Beijing (18\% of the fleet), China

93 over a period of three weeks (March 2 to 25, 2009). It includes a total of 255 million data points

94 representing $3.4 \times 10^{7}$ miles of travel and over two million trips. Each data point contains a timestamp up

95 to seconds of when the data was recorded, vehicle ID, vehicle location at the recording time (longitude and latitude), and vehicle speed. We define a "trip" as vehicle traveling between two stop events at which 
the vehicle completely stops or idles (with speed less than $1 \mathrm{mph}$ ) for at least five minutes. Setting a minimum "dwell time" threshold is commonly used for trip separation in processing trajectory data passively collected by GPS devices [23]. The 5-minute threshold is selected to reflect the long signal light waiting time in Beijing and reduce false positive trip separations when vehicles stop at traffic lights or idle in traffic. Although trip start/end time and locations need to be inferred from the data, substantial agreement exist between GPS-derived travel patterns and self-reported ones [24]. Using a different threshold (e.g. 3 minutes) changes the parameters slightly but does not change the conclusions of this study. We also identify a home location for each vehicle as the location where it parks consistently for the greatest amount of time. Different vehicles can have different data sampling intervals, but the sampling interval for the same vehicle stays the same throughout the three weeks.

\subsection{Background on Beijing taxis}

Taxis in Beijing do not work for dispatch centers. Instead, they mainly provide hail services, which mean that taxis cruise along the streets and look for clients who signal their needs for taxis. The vehicles are owned by the taxi companies, along with vehicle plates and taxi licenses. Taxi drivers pay a fixed monthly fee to the taxi company for the right to operate. Each driver has the same vehicle during his or her contract time with the taxi companies and is responsible for any associated costs (e.g. fuel, maintenance, and upgrade). The driver possesses the vehicle $24 / 7$ and normally parks it at where he/she lives when off work. These properties make Beijing taxis share some characteristics with private vehicles (e.g. parking at home at night, routine trips leaving and returning home).

\subsection{Radius of gyration $\left(r_{g}\right)$}

González et al. (2008) suggested that human travel trajectories can be characterized by a timeindependent characteristic travel distance -- radius of gyration $\left(r_{g}\right)$ [10]. Although the method was developed using individual travel data captured through mobile phone traces which have lower resolution compared to trajectory data collected by GPS, the concepts can be generalized. We modify this approach 
121 to analyze taxi travel by taking into account the time a vehicle spends at each location. Because data

122 sampling intervals are not the same for all vehicles in our dataset, using the time-weighted $r_{g}$ can reduce

123 the bias due to data sampling frequency variances. In particular, the $r_{g}$ of vehicle $i$ is calculated using Eq.

124 1. By using the time (in seconds) a vehicle spent at a particular location as the weight, it is equivalent to

125 as if the GPS device is collecting data at every second. Therefore, although equation 1 includes a term $t$,

126 the unit of $r_{g}$ calculated is still miles.

$127 \quad r_{g(i)}=\sqrt{\frac{1}{n_{i}} \sum_{j=1}^{n_{i}} t_{i j}\left(r_{i j}-r_{c(i)}\right)^{2}}$

128 where $r_{g(i)}$ represents the radius of gyration for vehicle $i, n_{i}$ is the total number of recorded locations for

129 vehicle $i ; j$ is the $j^{\text {th }}$ reported location; $t_{i j}$ is the time (in seconds) vehicle $i$ spent at the $j^{\text {th }}$ location; $r_{i j}$ is

130 the position vector recorded for vehicle $i$ at the $j^{\text {th }}$ location; and $r_{c(i)}=\frac{1}{n_{i}} \sum_{j=1}^{n_{i}} t_{i j} r_{i j}$ is the "center of

131 mass" of the trajectories of vehicle $i$, representing the mean location of vehicle $i$. We use the Manhattan

132 distance in this study to provide better estimates for the actual travel distance between two locations in a

133 road network.

\section{2.4. Trajectory transformation}

135 To compare the trajectories of taxis with different spatial coverage and characteristic travel distances, taxi

136 trajectories can be rescaled and re-centered based on each individual vehicle's intrinsic reference frame

137 and $r_{g}$. Using its center of mass as the origin, each individual vehicle's intrinsic reference frame is rotated

138 with the moment of inertia and then rescaled with the standard deviation of the locations on each axis [10].

139 Similar to the calculation of $r_{g}$, we include the time spent at each location as weights. The transformation

140 process is described in Eq. 2 to 7 with more details in [10]:

$141 \quad I=\left(\begin{array}{l}I_{x x} I_{x y} \\ I_{y x} I_{y y}\end{array}\right)$ 
$142 \quad I_{x x}=\sum_{j=1}^{n_{i}} t_{i j} y_{i j}^{2}$

$143 \quad I_{y y}=\sum_{j=1}^{n_{i}} t_{i j} x_{i j}^{2}$

$144 \quad I_{x y}=I_{y x}=\sum_{j=1}^{n_{i}} t_{i j} x_{i j} y_{i j}$

$145 \cos \left(\theta_{i}\right)=-I_{x y}\left(\frac{1}{2} I_{x x}-\frac{1}{2} I_{y y}+\frac{1}{2} \mu\right)^{-1} \frac{1}{\sqrt{1+\frac{I_{x y}{ }^{2}}{\left(\frac{1}{2} I_{x x}-\frac{1}{2} I y y+\frac{1}{2} \mu\right)^{2}}}}$

146 with $\mu=\sqrt{4 I_{x y} I_{y x}+I_{x x}^{2}-2 I_{x x} I_{y y}+I_{y y}^{2}}$

147 where $I$ is the tensor of inertia; $t_{i j}$ is the time (in seconds) vehicle $i$ spent at the $j^{\text {th }}$ location; $x_{i j}$ is the

148 longitude recorded for vehicle $i$ at the $j^{\text {th }}$ location; $y_{i j}$ is the latitude recorded for vehicle $i$ at the $j^{\text {th }}$

149 location; and $\theta_{i}$ is the angle of rotation to transform the trajectories of vehicle $i$ to its intrinsic reference

150 frame.

151

To ensure comparativeness and consistency, the most frequently visited location is ensured to be

152 on the positive $x$-axis by imposing an additional $180^{\circ}$ rotation for vehicles with the most frequently

153 visited locations laying in $x_{i j}<0$.

154 To compare trajectories at different spatial scales, the rotated trajectories $\left(x^{\prime}, y^{\prime}\right)$ are then 155 rescaled with the standard deviation of the locations as $\left(x^{\prime} / \sigma_{x}, y^{\prime} / \sigma_{y}\right)$ using Eq. 8 and 9.

$156 \quad \sigma_{x(i)}=\sqrt{\frac{1}{n_{i}} \sum_{j=1}^{n_{i}} t_{i j}\left(x_{i j}-x_{c(i)}\right)^{2}}$

$157 \quad \sigma_{y(i)}=\sqrt{\frac{1}{n_{i}} \sum_{j=1}^{n_{i}} t_{i j}\left(y_{i j}-y_{c(i)}\right)^{2}}$ 
where $\sigma_{x(i)}$ is the standard deviation on x-axis for vehicle $i ; \sigma_{y(i)}$ is the standard deviation on y-axis for

159 vehicle $i ; x_{c(i)}=\frac{1}{n_{i}} \sum_{j=1}^{n_{i}} t_{i j} x_{i j}$ is the x-axis value of the "center of mass" of the trajectories of vehicle $i$;

160 and $y_{c(i)}=\frac{1}{n_{i}} \sum_{j=1}^{n_{i}} t_{i j} y_{i j}$ is the y-axis value of the "center of mass" of the trajectories of vehicle $i$.

\section{Results and discussion}

\subsection{Characteristic travel distance and mass center}

163 Strong spatiotemporal regularities exist for taxi trajectories. On a daily basis, taxi drivers leave home in 164 the morning for business and return home at late night. This routine could be easily identified from the segments of flat lines in Figure 1 showing the periods of time when this example taxi is parked at its driver's home (taxi drivers in Beijing normally take the vehicle home after work, please refer to the

167 Method section for more background information about the taxi fleet in Beijing).

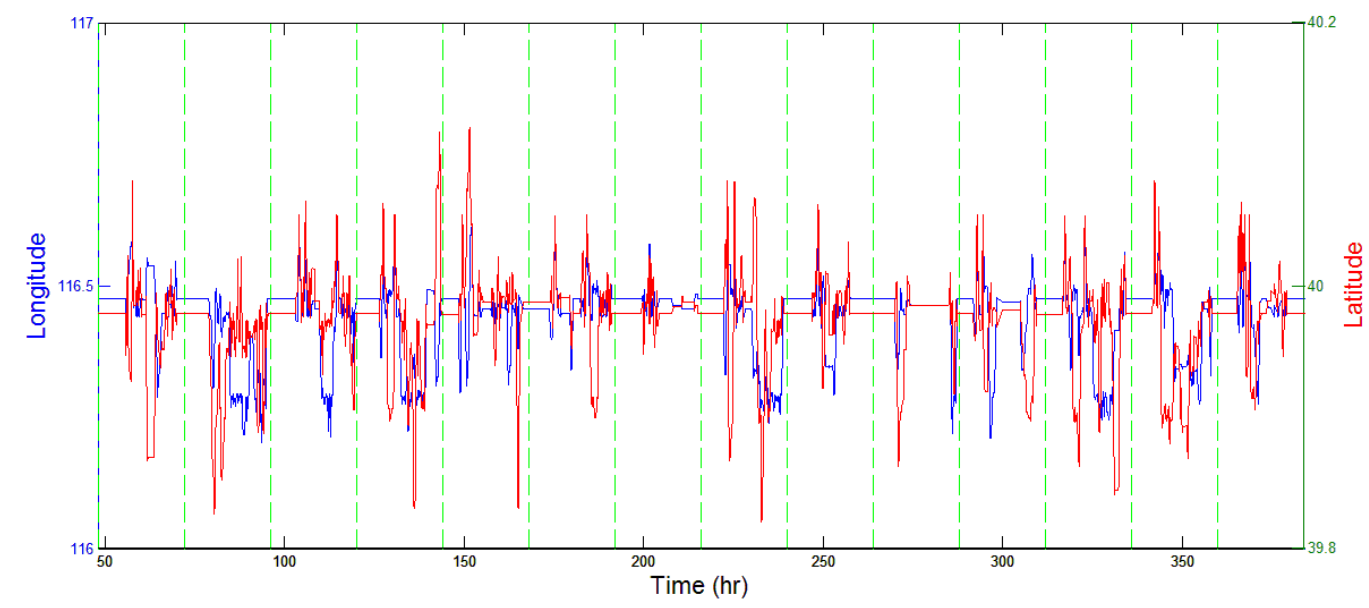

Figure 1. Longitude and latitude over time for a typical taxi. Longitude is plotted in blue using left-handside y-axis, while latitude is plotted in red using right-hand-side y-axis. Green dotted line shows the

171 midnight of a day. 
173 centers of mass (mean locations, $r_{c(i)}$ ) are highly skewed towards the city center. While taxi drivers live 174 in the city and its vicinity (Figure 2a), the mass centers of taxi trajectories are highly concentrated in the 175 inner city (Figure 2b). This could be explained by the fact that while residential locations expanded with 176 urban sprawl, major functions of the city are still concentrated in the central area [25]. The distribution of 177 distances between home locations and the centers of mass follow an exponential decay (Figure 2c), 178 indicating the significant impact of home location in determining the center of mass. For $98 \%$ of the taxis, 179 the distance between the home location and the mass center is less than or equal to 10 miles.

The radius of gyration $\left(r_{g}\right)$ describes the spatial coverage of a taxi, indicating whether the trajectory of the taxi is concentrated in a small area or more spread out. The distribution of $r_{g}$ for all taxis 182 does not follow a truncated power-law distribution as observed in previous studies [10, 26], possibly due 183 to the fact that taxi travels are the result of a combination of driver's commuting needs, passenger's travel needs, and driver's cruise between passenger trips. Compared to the truncated power-law fit, the probability of taxis to have small $r_{g}$ is significantly lower. Only $1.5 \%$ of taxis in this dataset have $r_{g}$ that is less than or equal to 3 miles. Activities of these taxis mainly concentrate in the city center. The home 187 locations of these taxis also tend to be close to the city center as well. Considering Tiananmen Square as 188 the center of Beijing, the average distance from home to the center of the city is only 12.9 miles for these 189 taxis. Approximately $93.5 \%$ of the taxis have $r_{g}$ between 3 and 20 miles. The probability of a taxi to have $190 r_{g}$ that is within this range is much higher than the probability obtained from the truncated power-law 191 distribution. About $0.1 \%$ of the taxis have $r_{g}$ greater than 100 miles mostly due to a few extremely long 192 trips that are probably prearranged business travel rentals or drivers' personal leisure travels. 

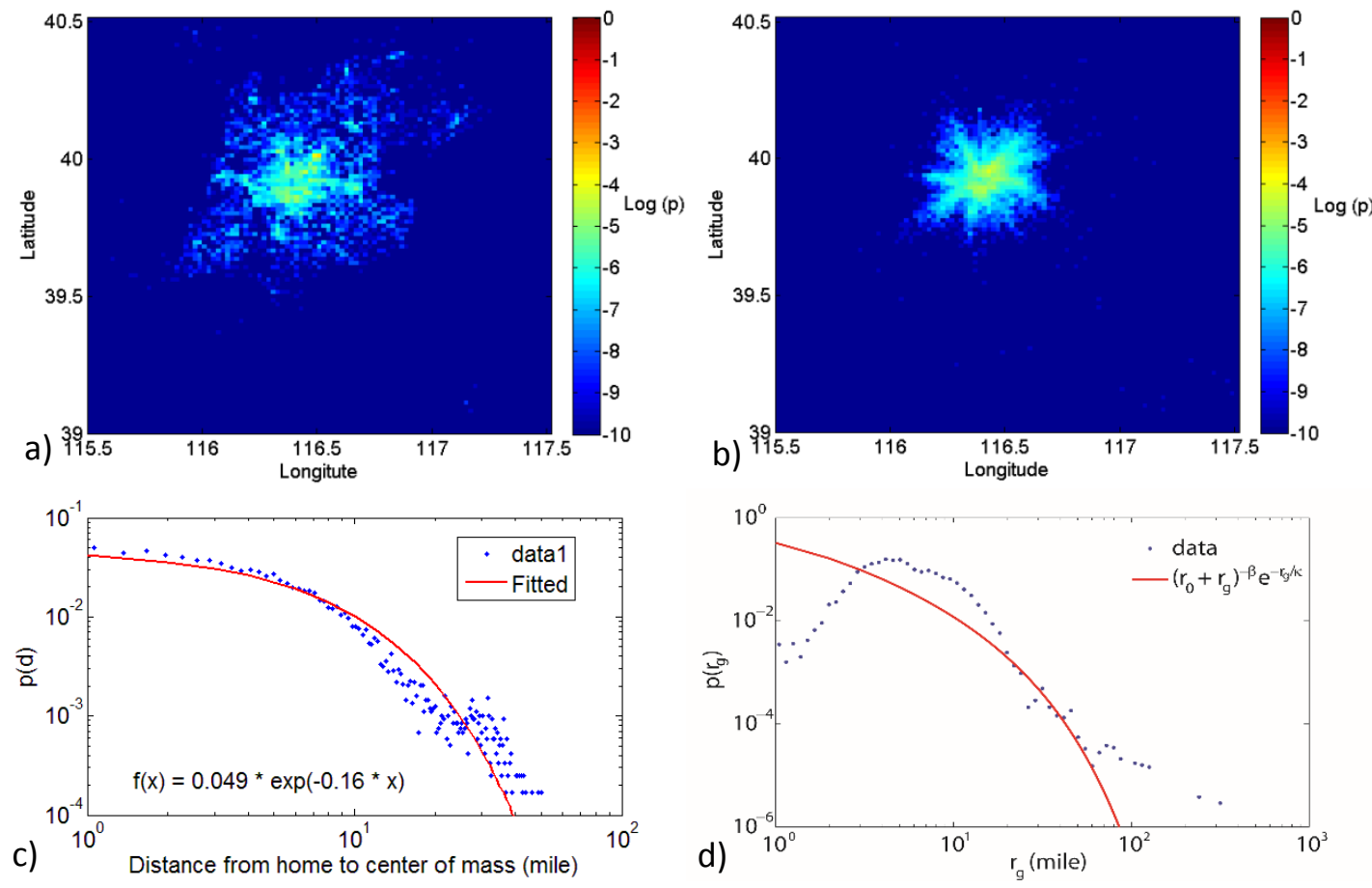

Figure 2. a) Spatial distribution of taxis home location; b) spatial distribution of taxis centers of mass (mean location); c) distribution of distance from home location to the center of mass following an exponential decay; and d) probability distribution of radius of gyration $\left(\mathrm{r}_{\mathrm{g}}\right)$ with $r_{0}=1$ mile, $\beta=1.5, \kappa=$ 12.

\subsection{Distribution of trip distances}

The distribution of trip distances is another important measure for travel patterns. Two models exist to describe trip distance distribution: the truncated power-law distribution and the exponential distribution. Gozález et al. (2008) found that truncated power-law in the form of $P(\Delta r)=\left(\Delta r+\Delta r_{0}\right)^{-\beta} \exp (-\Delta r / \kappa)$ can well approximate individual trip displacement for individual people using cell phone traces, with $\beta=1.75 \pm 0.15, \Delta r_{0}=1.5 \mathrm{~km}$, and $\kappa=80 \sim 400 \mathrm{~km}[10]$. The scaling of a truncated power law means that the probability of taking long trips is non-negligible. Liang et al. (2012) argued that the displacement of taxi passenger trips follows an exponential decay with exponent $\lambda=0.1689 \sim 0.2329$, indicating that the likelihood of large displacement trip decreases exponentially and is negligible [17]. We use both 
models to fit the trip displacements observed in this study. Our results show that the tail of our data falls between these two fitted models (Figure 3a). Model parameters estimated in this research are in the similar range with aforementioned studies. This indicates that the probability of taxis taking long distance trips ( $d \geq 30$ miles) is not as significant as what a power-law distribution predicts, but is greater than what an exponential decay implies. We verified that the distribution of the empirical data is statistically greater than the fitted exponential distribution and smaller than the fitted truncated power-law distribution 214 ( $\mathrm{p}$-value $=0.665$, details provided in the Supplemental Information). Based on the observed differences, we further fitted the probability distribution of short trips $(d<30$ miles $)$ and long trips $(d \geq 30$ miles $)$ separately and found that the distribution of short trips follows power law while the distribution of long trips is exponential (Figure 3b, p-value $=0.309$, details provided in the Supplemental Information). a)

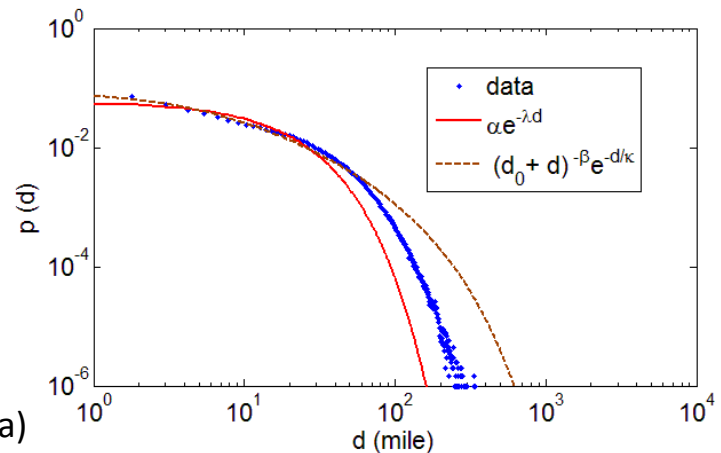

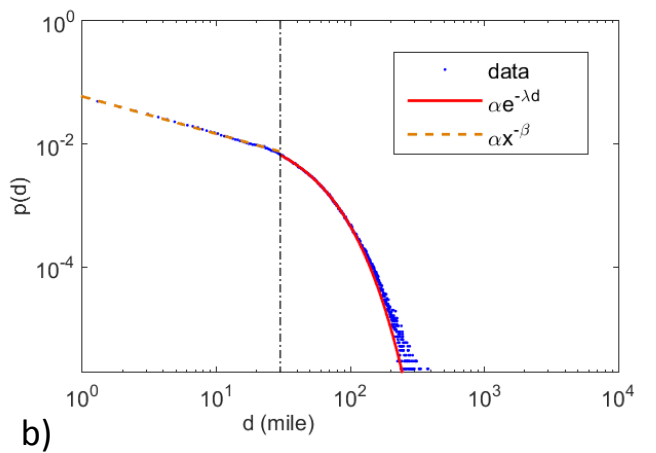

b)

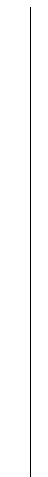

Figure 3. The probability density function $p(d)$ of travel distance $d$ fitted with different models. a) the entire curve is fitted with two previously identified models: 1) exponential distribution with $\alpha=0.06$, $\lambda=0.068$; and 2) truncated power-law distribution with $d_{0}=6.9$ miles, $\beta=1.254$, and $\kappa=$ 107.6 miles. b) short trips ( $\mathrm{d}<30$ miles) and long trips ( $\mathrm{d} \geq 30$ miles) are fitted separately: the short trips can be best fitted with a power-law distribution with $\alpha=0.06, \beta=0.061$; and the long trips can be best fitted with an exponential distribution with $\alpha=0.02, \lambda=0.038$.

\section{3.3. Spatial density distribution}

226 The normalized spatial density distribution represents the probability of finding a taxi at a location in its

227 intrinsic reference frame (Figure 4). When $r_{g}$ is less than 10 miles, similar spatial distribution is observed 
228 with those obtained using individual mobile phone traces and private vehicle trajectories [10, 27]. It is

229 more likely for taxis to travel to places that are closer to their centers of mass and less likely to visit

230 places that are farther away. However, when $r_{g}$ increases, slightly different patterns emerge (Figure 5a).

231 Instead of having one location with high probability (Figure $5 b$ ), vehicles with higher $r_{g}$ have two separate

232 high-probability locations (Figure 5c). To identify what has caused the multiple high-probability locations,

233 we separated the trajectories of occupied trips and unoccupied trips and plotted the spatial density

234 distribution separately. The emergence of two high-probability locations is only observed in unoccupied

235 trips but not in occupied trips (Figure 6), which further confirmed the difference between trajectories of

236 these two types of trips and the importance of including unoccupied trips to study taxi travel patterns.

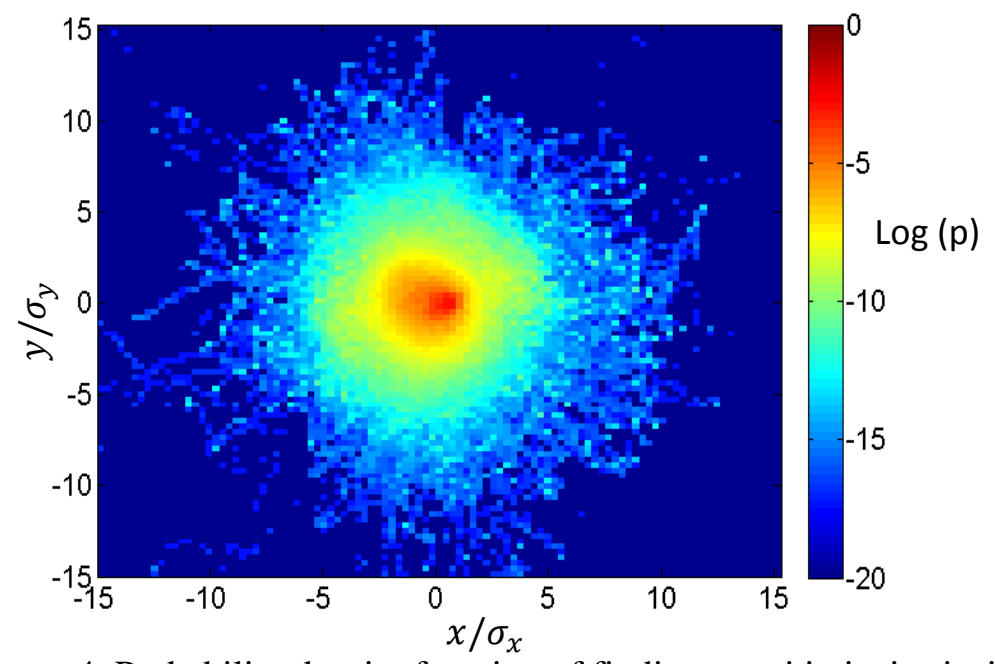

Figure 4. Probability density function of finding a taxi in its intrinsic reference frame, for taxis whose $r_{g}$ is

239 greater than 4 miles but less than 5 miles. 

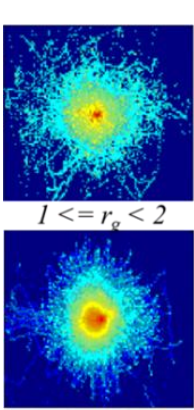

$5<=r_{g}<6$

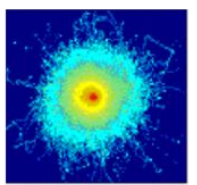

$2<=r_{\alpha}<3$

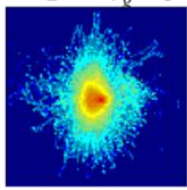

$6<=r_{g}<7$
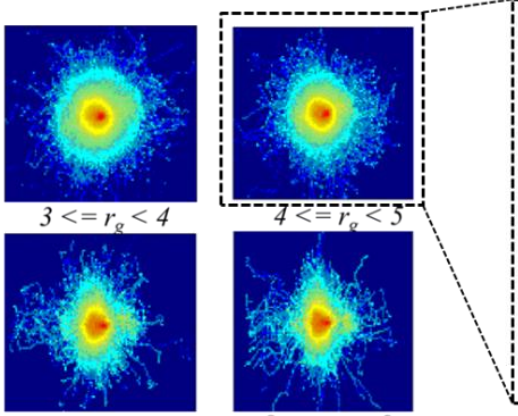

b)
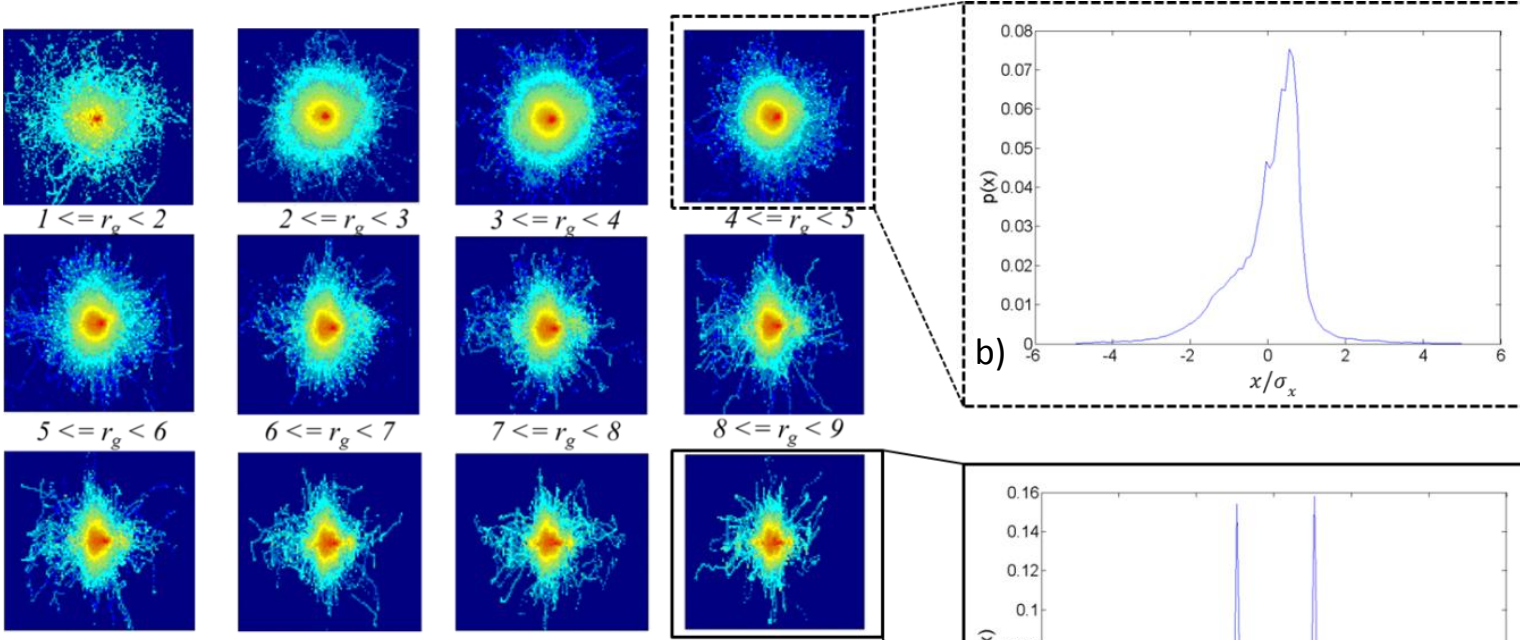

$9<=r_{8}<10$

$10<=r<11$

$11<=r_{8}<12$
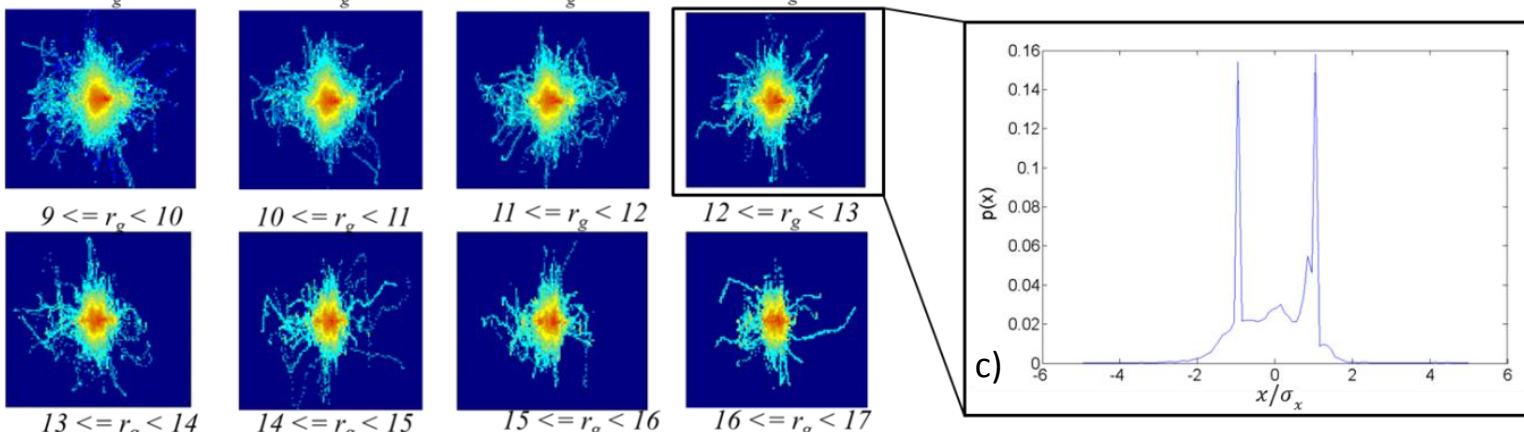

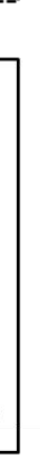

241 Figure 5. a) Probability density function of finding a taxi in its intrinsic reference frame; b) probability

242 density function projected onto one dimension for vehicles with $r_{g}$ between 4 and 5 miles; and c)

243 probability density function projected onto one dimension for vehicles with $\mathrm{r}_{\mathrm{g}}$ between 12 and 13 miles.

\section{Unoccupied trips}

a)

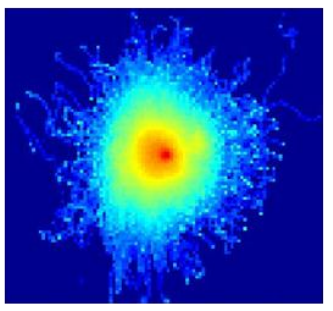

$$
4<=r_{g}<5
$$

Occupied trips

b)

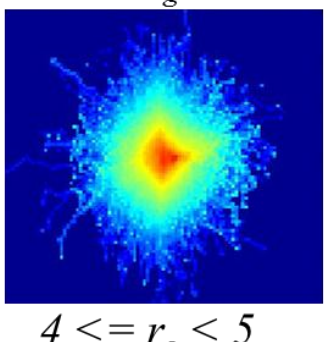

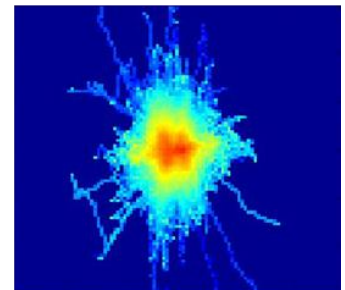

$11<=r_{g}<12$

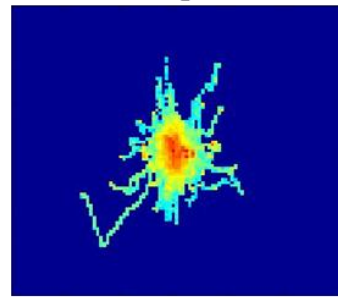

$11<=r_{g}<12$

Figure 6. Probability density function of finding a taxi in its intrinsic reference frame for selected $r_{g}$ range for a) trajectories of unoccupied trips and b) trajectories of occupied trips. 


\section{4. Conclusions}

248 In this paper, we examine the travel patterns of taxis from a holistic vehicle travel perspective, which

249 considers not only the trips to satisfy passenger needs, but also the movement of taxis to satisfy the travel

250 needs of the drivers (e.g. leaving from home for business and returning to home after work, cruising along

251 streets for customers, personal travels). Our results show that taxi travel patterns share similar traits with

252 travel patterns of individuals, but also exhibit specific differences. Trip displacement distribution of taxi

253 travels is statistically greater than the exponential distribution and smaller than the truncated power-law

254 distribution. The distribution of short trips (less than 30 miles) can be best fitted with power-law while

255 long trips (equals to or greater than 30 miles) follow exponential decay. Characteristic travel distance

256 does not follow a truncated power-law as observed in previous studies which only examined passenger

257 trips. Spatial and temporal regularities exist in taxi travels when the trajectories are transformed into each

258 vehicle's intrinsic reference frame. However, with increasing spatial coverage, taxis with greater

259 characteristic travel distance appear to have dual high-probability locations.

These results show that characteristics of passenger taxi trips cannot be used to represent the

vehicular travels of taxis. It is important to include non-occupied trips to fully understand the travel

262 patterns of taxis from the vehicular travel perspective. Results from this study can inform taxi travel

263 modeling (e.g. trip generation, agent-based modeling) and improve transportation system analysis (e.g.

264 electric taxi adoption and charging behavior, charging infrastructure siting, taxi ride sharing). It is worth

265 noting that urban form, infrastructure and taxi regulations can impact taxi travels in different cities. While

266 we expect that the importance of holistically analyzing taxi trips persists, further research using data from

267 multiple cities is needed to examine to what extend the specific results observed in this study can be

268 generalized. The methods developed in this study can be applied to analyze data from other cities. 


\section{Acknowledgements}

This material is based upon work partially supported by the Department of Energy under Award Number DE-PI0000012. H.C. also acknowledges the support from the ERM Group Foundation.

\section{References}

[1] L. Mari, E. Bertuzzo, L. Righetto, R. Casagrandi, M. Gatto, I. Rodriguez-Iturbe, A. Rinaldo, Modelling cholera epidemics: the role of waterways, human mobility and sanitation, Journal of The Royal Society Interface, 9 (2012) 376-388.

[2] L. Hufnagel, D. Brockmann, T. Geisel, Forecast and control of epidemics in a globalized world, Proceedings of the National Academy of Sciences of the United States of America, 101 (2004) 1512415129.

[3] B. Jiang, J. Yin, S. Zhao, Characterizing the human mobility pattern in a large street network, Physical Review E, 80 (2009) 021136.

[4] R. Kitamura, C. Chen, R. Pendyala, R. Narayanan, Micro-simulation of daily activity-travel patterns for travel demand forecasting, Transportation, 27 (2000) 25-51.

[5] H.D. Rozenfeld, D. Rybski, J.S. Andrade, M. Batty, H.E. Stanley, H.A. Makse, Laws of population growth, Proceedings of the National Academy of Sciences, 105 (2008) 18702-18707.

[6] C. Nash, Genetics, Race, and Relatedness: Human Mobility and Human Diversity in the Genographic Project, Annals of the Association of American Geographers, 102 (2011) 667-684.

[7] Y. Yue, T. Lan, A.G.O. Yeh, Q.-Q. Li, Zooming into individuals to understand the collective: A review of trajectory-based travel behaviour studies, Travel Behaviour and Society, 1 (2014) 69-78.

[8] D. Brockmann, Hufnagel, L., Geisel, T., The scaling laws of human travel, Nature, 439 (2006) 4.

[9] R. Injong, S. Minsu, H. Seongik, L. Kyunghan, K. Seong Joon, C. Song, On the Levy-Walk Nature of Human Mobility, Networking, IEEE/ACM Transactions on, 19 (2011) 630-643.

[10] C.A.H. Marta C. González, and Albert-László Barabási, Understanding individual human mobility patterns, Nature, 453 (2008) 779-782.

[11] C. Song, T. Koren, P. Wang, A.-L. Barabasi, Modelling the scaling properties of human mobility, Nature Physics, 6 (2010) 6.

[12] X.-Y. Yan, Han, Xiao-Pu, Wang, Bing-Hong, Zhou, Tao, Diversity of individual mobility patterns and emergence of aggregated scaling laws, Scientific Reports, 3 (2013).

[13] J.M. Salanova, M. Estrada, G. Aifadopoulou, E. Mitsakis, A review of the modeling of taxi services, Procedia - Social and Behavioral Sciences, 20 (2011) 150-161.

[14] C. Roth, S.M. Kang, M. Batty, M. Barthélemy, Structure of Urban Movements: Polycentric Activity and Entangled Hierarchical Flows, PLoS ONE, 6 (2011) e15923.

[15] X. Liu, L. Gong, Y. Gong, Y. Liu, Revealing travel patterns and city structure with taxi trip data, Journal of Transport Geography, 43 (2015) 78-90.

[16] C. Peng, X. Jin, K.-C. Wong, M. Shi, P. Liò, Collective Human Mobility Pattern from Taxi Trips in Urban Area, PLoS ONE, 7 (2012) e34487.

[17] X. Liang, X. Zheng, W. Lv, T. Zhu, K. Xu, The scaling of human mobility by taxis is exponential, Physica A: Statistical Mechanics and its Applications, 391 (2012) 2135-2144. 
[18] J. Yuan, Y. Zheng, L. Zhang, X. Xie, G. Sun, Where to find my next passenger, in: Proceedings of the 13th international conference on Ubiquitous computing, ACM, Beijing, China, 2011, pp. 109-118.

310 [19] L. Liu, C. Andris, C. Ratti, Uncovering cabdrivers' behavior patterns from their digital traces,

311 Computers, Environment and Urban Systems, 34 (2010) 541-548.

312 [20] D. He, H. Liu, K. He, F. Meng, Y. Jiang, M. Wang, J. Zhou, P. Calthorpe, J. Guo, Z. Yao, Q. Wang,

313 Energy use of, and CO2 emissions from China's urban passenger transportation sector - Carbon

314 mitigation scenarios upon the transportation mode choices, Transportation Research Part A: Policy and

315 Practice, 53 (2013) 53-67.

316 [21] A. Krieger, L. Wang, P. Radtke, C. Malorny, Recharging China's electric vehicle aspirations: A

317 perspective on revitalizing China's electric vehicle industry, in: McKinsey \& Company Report, 2012.

318 [22] BBC, New London taxis to emit zero emissions from 2018, in: BBC News, 2014.

319 [23] J. Liu, H. Zheng, T. Feng, S. Yuan, H. Lu, Post-processing Procedures for Passive GPS based Travel

320 Survey, Procedia - Social and Behavioral Sciences, 96 (2013) 310-319.

321 [24] D. Houston, T.T. Luong, M.G. Boarnet, Tracking daily travel; Assessing discrepancies between

322 GPS-derived and self-reported travel patterns, Transportation Research Part C: Emerging Technologies,

32348 (2014) 97-108.

324 [25] P. Zhao, Sustainable urban expansion and transportation in a growing megacity: Consequences of 325 urban sprawl for mobility on the urban fringe of Beijing, Habitat International, 34 (2010) 236-243.

326 [26] L. Pappalardo, S. Rinzivillo, Z. Qu, D. Pedreschi, F. Giannotti, Understanding the patterns of car 327 travel, Eur. Phys. J. Spec. Top., 215 (2013) 61-73.

328 [27] F. Giannotti, M. Nanni, D. Pedreschi, F. Pinelli, C. Renso, S. Rinzivillo, R. Trasarti, Unveiling the 329 complexity of human mobility by querying and mining massive trajectory data, The VLDB Journal, 20 330 (2011) 695-719. 